\title{
Quantitative assessment of fluorescence intensity of ICG in sentinel nodes in early gastric cancer
}

\author{
Keishi Okubo ${ }^{1}$. Yoshikazu Uenosono ${ }^{1,2} \cdot$ Takaaki Arigami $^{1,2} \cdot$ Daisuke Matsushita $^{1} \cdot$ Shigehiro Yanagita $^{1}$. \\ Takashi Kijima $^{1} \cdot$ Masahiko Amatatsu $^{1} \cdot$ Sumiya Ishigami ${ }^{1} \cdot$ Kosei Maemura $^{1} \cdot$ Shoji Natsugoe $^{1,2}$
}

Received: 10 May 2017 / Accepted: 27 February 2018 / Published online: 7 March 2018

(c) The International Gastric Cancer Association and The Japanese Gastric Cancer Association 2018

\begin{abstract}
Background The sentinel node (SN) detection by dual tracer method using indocyanine green (ICG) and a radioisotope (RI) has been recommended for early gastric cancer. However, institutions are limited due to radioactivity in the RI method. The greatest advantage of the RI method is that it objectively assesses RI uptake as a numerical value. The aim of the present study was to verify the usefulness of ICG fluorescence intensity in SN.

Methods Seventeen patients with early gastric cancer were enrolled in this study. RI uptake by each lymph node was measured using Navigator GPS and fluorescence nodes were identified using the hyper eye medical system (HEMS). Fluorescence intensity in fluorescence nodes was evaluated using ICG intensity imaging software (Mizuho, Japan) of the HEMS.

Results The total number of dissected lymph nodes was 227, with an average of 13.3 per patient. The numbers of HN, FN-S, and FN-B were 64,77 , and 34. RI uptake was significantly greater by FN-S than by non-FN-S $(P=0.0016)$. The median fluorescence intensity value was higher in $\mathrm{HN}$ than in non-HN $(P<0.001)$. A correlation was observed between RI uptake and fluorescence intensity. Dissecting FNs with fluorescence intensity levels of 1-6 resulted in $92.1 \%$ dissection of HNs.

Conclusion It is possible that the evaluation of fluorescence intensity is useful for selected SNs instead of RI tracer. If fluorescence intensity is measurable in surgery, an infrared fluorescence method using ICG may be useful and safe for the detection of SN in early gastric cancer.
\end{abstract}

Keywords Gastric cancer · Sentinel node navigation surgery $\cdot$ Indocyanine green $\cdot$ Near-infrared fluorescence imaging . Fluorescence intensity

\section{Introduction}

Since gastrectomy with D2 lymph node dissection became the standard procedure for gastric cancer worldwide, its prognosis has improved [2]. Gastric cancer is now

Electronic supplementary material The online version of this article (https://doi.org/10.1007/s10120-018-0816-z) contains supplementary material, which is available to authorized users.

Keishi Okubo

ok0627@m2.kufm.kagoshima-u.ac.jp

1 Department of Digestive Surgery, Breast and Thyroid Surgery, Kagoshima University Graduate School of Medical and Dental Sciences, 8-35-1 Sakuragaoka, Kagoshima 890-8520, Japan

2 Molecular Frontier Surgery, Course of Advanced Therapeutics, Kagoshima University Graduate School of Medical and Dental Sciences, Kagoshima, Japan discovered in the early stages in many cases due to recent advances in endoscopic diagnoses. Endoscopic mucosal resection (EMR) and endoscopic submucosal dissection (ESD) are currently only performed for early gastric tumors (cT1 and N0); however, standard gastrectomy and lymphadenectomy are recommended for histologically undifferentiated-type adenocarcinomas and submucosal tumors. The actual rate of lymph node metastasis ranges between 5 and 10\% among patients with cT1 gastric cancer. Accordingly, many patients without lymph node metastasis undergo unnecessary lymphadenectomy.

In several facilities, the concept of the sentinel node (SN) was analyzed in order to prove that sentinel node navigation surgery (SNNS) is a safe and minimally invasive surgery for patients who need to undergo standard lymphadenectomy. The sentinel lymph node concept has been established in various cancers to avoid unnecessary lymphadenectomy $[5,14]$. The sentinel lymph node concept for gastric cancer 
was initially suggested in studies from Japan in 2000 [1, 6, 9]. We have been using radioisotope methods since 2000 to investigate SNNS for gastric cancer $[4,19]$. We reported that $\mathrm{SN}$ were detected in $78(99 \%)$ out of 79 patients with cT1N0 tumors by $99 \mathrm{mTc}$-radiolabeled tin colloid methods, with sensitivity and accuracy rates of $100 \%$ [20]. We also demonstrated the clinical usefulness of the rapid RT-PCR system to detect sentinel metastasis in gastric cancer [22].

A prospective multicenter trial on SN mapping for gastric cancer (cT1 and T2) in Japan showed that SNNS with basic $\mathrm{RI}$ methods is a feasible procedure. In this study, basin dissection by the dual tracer of RI and dye-guided methods is safe because only four false-negative $\mathrm{SN}$ biopsies were obtained [10]. Furthermore, lymph node metastases, including micrometastases, need to be detected by histopathological and molecular biological analyses for SNNS [4, 18, 21].

The indocyanine green (ICG) method has been suggested to be useful for detecting the SNs in combination with infrared electronic endoscopy $[7,11,16]$. Recent studies reported the development and usefulness of ICG near-infrared fluorescence images [12,23]. The RI method is useful in that RI uptake in the SNs can be counted with a gamma probe and the count reflects the amount of lymph flow. Therefore, the RI method is useful for selecting SN for intraoperative histological examinations using hematoxylin and eosin (HE) staining. However, institutions are limited due to radioactivity in the RI method. On the other hand, a quantitative evaluation of ICG fluorescence has not yet been conducted. If it is possible to evaluate the fluorescence intensity of ICG uptake using ICG fluorescence imaging, ICG methods in SNNS may be conducted even in hospitals without special RI equipment.

In the present study, we investigated the relationship between RI uptake and ICG fluorescence intensity in SNNS for early gastric cancer and confirmed the utility of ICG fluorescence methods.

\section{Materials and methods}

\section{Patients}

Between February 2013 and August 2015 in the Department of Digestive Surgery of Kagoshima University, 17 patients with a preoperative diagnosis of cT1-2 and cN0 early gastric cancer were enrolled in this study (Table 1). Patients with early gastric cancer less than $4 \mathrm{~cm}$ in diameter that was confined to the mucosa or submucosa without obvious metastasis were included in the present study. Informed consent was obtained from all patients based on a document approved by the Ethics Committee of the hospital. We conducted computed tomography, endoscopy, a barium study, endoscopic
Table 1 Patient backgrounds

\begin{tabular}{ll}
\hline Surgery & \\
LADG & 4 \\
LAPG & 1 \\
LPG & 12 \\
Tumor size & $19.6 \mathrm{~mm}$ \\
& \multicolumn{2}{c}{$(6-40 \mathrm{~mm})$} \\
Location & \\
U & 5 \\
M & 10 \\
L & 2 \\
cT factor & \\
1 & 15 \\
2 & 2 \\
Pathological type \\
Differ & 8 \\
Undiffer & 9 \\
\hline
\end{tabular}

ultrasonography, and abdominal echo on all patients in order to diagnose the preoperative stage.

Fifteen patients had cT1 tumors, while 2 had cT2 tumors. Twelve patients underwent laparoscopic partial gastrectomy (LPG), 4 laparoscopic assistant distal gastrectomy (LADG), and 1 laparoscopic pylorus gastrectomy (LAPG) (Supplementary Table 1).

\section{Lymphatic mapping technique}

One day before surgery, $99 \mathrm{~m}$-Technetium-Tin colloid and ICG were endoscopically injected in every case. We mixed $99 \mathrm{~m}$-Technetium-Tin colloid with ICG (200-fold dilution) in a total volume of $3 \mathrm{ml}(4.5 \mathrm{mCi})$. Subsequently, $0.5 \mathrm{ml}$ each of a mixture of ${ }^{99 \mathrm{~m}}$ Technetium-Tin colloid ( $3 \mathrm{mCi}$ ) and ICG was injected submucosally at four sites around the tumor. During surgery, RI uptake of each lymph node was measured using Navigator GPS (Tyco Healthcare, Ltd., Tokyo Japan) and ICG fluorescence imaging were identified by an Infra Red Imaging (IRI) system (OLYMPUS). The total number of dissected lymph nodes was 227 , and RI uptake or ICG fluorescence intensity was investigated in 111 . All dissected SN were examined by H\&E staining.

\section{Evaluation of hot nodes (HN) and fluorescence nodes (FN)}

When counts for individual lymph nodes by Navigator GPS were 10-fold greater than background levels, the lymph node was identified as a "hot node" (HN) [19] and SN. Fluorescence nodes (FNs) were also identified by a hyper eye medical system (HEMS, Mizuho Corporation, Ltd., Tokyo Japan). Lymph nodes identified by the IRI System during surgery were defined as surgical fluorescence nodes (FN-S) (Fig. 1), and marked using clips. Lymph nodes with ICG 
Fig. $1 \mathrm{LN}$ vessels and $\mathrm{LN}$ are clearly visualized by IREE (infrared ray electronic endoscopes) with indocyanine green (ICG). a White view observation of LN. b Infrared ray observation of fluorescence lymph nodes
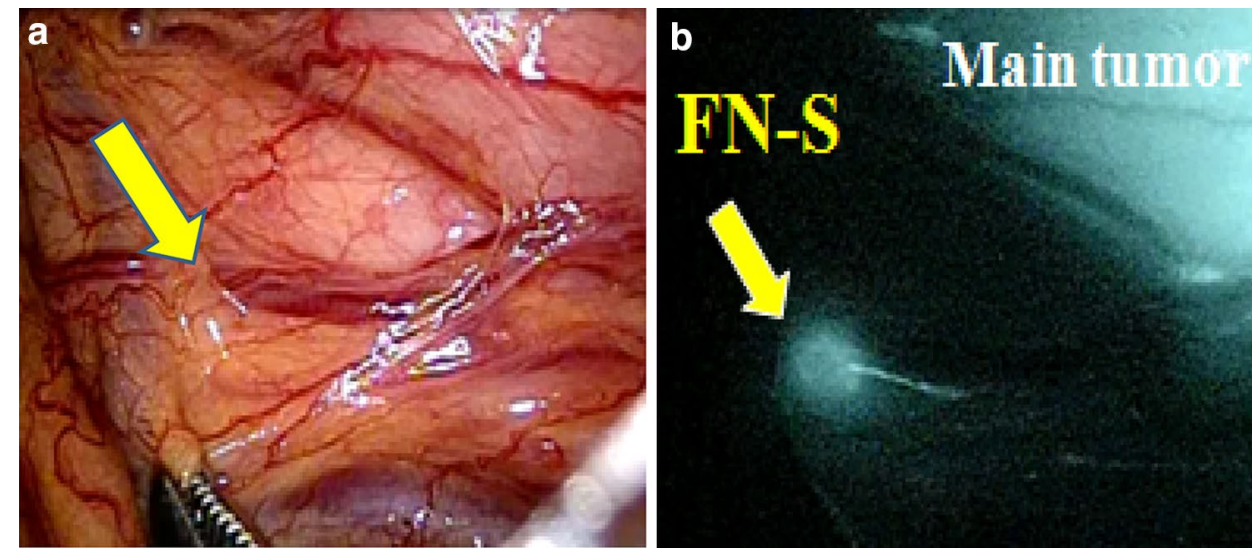

Fig. 2 Evaluation of fluorescence intensity by ICG intensity imaging software (Mizuho, Japan). Fluorescence intensity was calculated as FN intensity subtracted by background intensity. a View of HEMS image. b View using ICG intensity imaging software
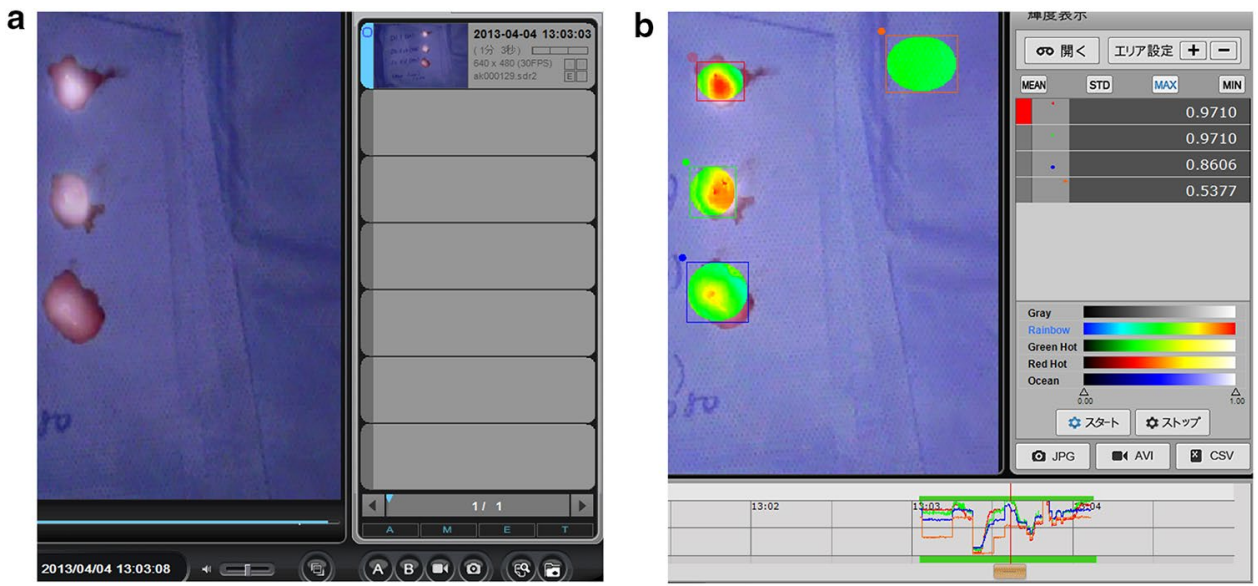

fluorescence detected by HEMS in the back table after basin nodal dissection except for FN-S were defined as FN-B. Therefore, FNs included both FN-S and FN-B. The RI and ICG uptake was measured for all FNs.

\section{Evaluation of fluorescence intensity}

The fluorescence intensities of all FN were evaluated using ICG intensity imaging software (Mizuho Corporation, Ltd., Tokyo, Japan) after surgery. Fluorescence intensity was calculated as background intensity subtracted by FN intensity (Fig. 2). The purpose of the present study was to investigate the relationship between RI uptake and fluorescence intensity from ICG.

\section{Statistical analysis}

All statistical calculations were performed using SAS/JMP statistical software (SAS Institute. Inc., Cary, NC). Clinicopathological variables were analyzed using Pearson's Chi squared test. A $P$ value of $<0.05$ was considered to be significant.
Table 2 Detected HN and FN

\begin{tabular}{lll}
\hline & $\begin{array}{l}\text { Total number of lymph } \\
\text { nodes }\end{array}$ & $\begin{array}{l}\text { Average number } \\
\text { of lymph nodes }\end{array}$ \\
\hline HN & 64 & $3.76(1-7)$ \\
FN-S & 77 & $4.52(1-7)$ \\
FN-B & 34 & $2.00(0-7)$ \\
ALL FNs & 111 & $6.52(3-17)$ \\
Dissected lymph nodes & 227 & $13.3(4-41)$ \\
\hline
\end{tabular}

\section{Results}

\section{Numbers of $\mathrm{HN}$ and FN}

$\mathrm{HN}$ and FN-S were identified in all 17 patients. All dissected lymph nodes were examined by $\mathrm{H} \& \mathrm{E}$ staining and metastasis was not detected in any lymph nodes. The total number of all dissected lymph nodes was 227 (mean 13.3). The numbers of HN, FNs, FN-S, and FN-B were 64 (mean 3.76), 111 (mean 6.52), 77 (mean 4.52), and 34 (mean 2.00 ), respectively (Table 2 ). RI uptake by the sentinel 
lymph node ranged between 10 and 731 counts per second (cps) (mean $63.7 \mathrm{cps}$ ). The total number of $\mathrm{HN}$ or FN-S was 88 , and the $\mathrm{HN}$-positive rate was $60.2 \%$ (53 out of 88). The $\mathrm{FN}-\mathrm{S}$ positive rate in $\mathrm{HN}$ was $82.8 \%$ (53 out of 64 ) and the HN only positive rate was $17.2 \%$ (11 out of 64) (Table 3). HN and FN-S lymph nodes accounted for $47.7 \%$ (53 of 111).

\section{Evaluation of RI uptake and fluorescence intensity}

RI uptake was significantly greater by FN-S $(77.5 \pm 14.9)$ than by FN-B $(32.5 \pm 10.8)(P=0.0016)$ (Fig. 3a). The median values for the fluorescence intensities of $\mathrm{HN}, \mathrm{FN}-\mathrm{S}$,

Table 3 Correlation between FN-S and HN

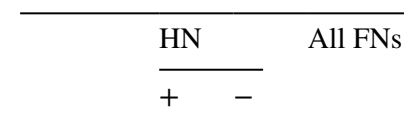

\begin{tabular}{lrrr}
\hline FN-S & & & \\
+ & 53 & 24 & 77 \\
- & 11 & 23 & 34 \\
All FNs & 64 & 47 & 111 \\
\hline
\end{tabular}

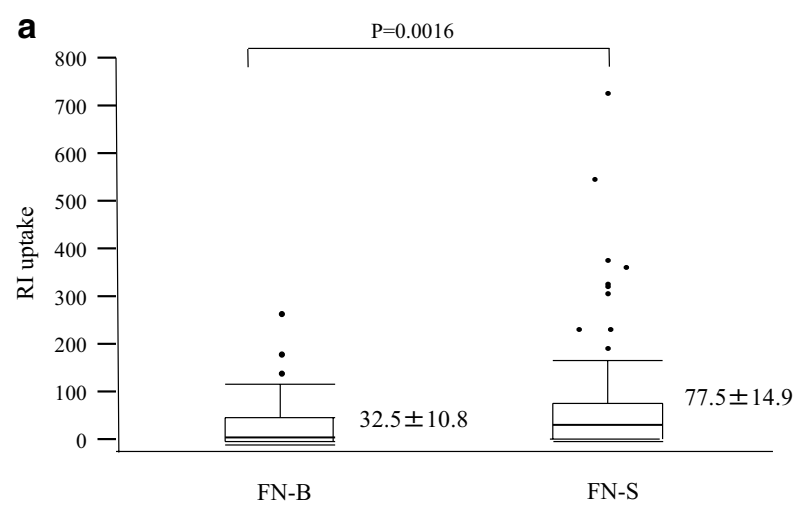

and FN-B were $0.414,0.386$, and 0.226 , respectively. The median values for the fluorescence intensities of $\mathrm{HN}$ and non-HN were $0.414 \pm 0.028$ and $0.228 \pm 0.034$, respectively. Fluorescence intensity was significantly higher in $\mathrm{HN}$ than in non-HN $(P<0.001)$ (Fig. 3b). The median values for the fluorescence intensities of FN-S and FN-B were $0.386 \pm 0.029$ and $0.226 \pm 0.030$. Fluorescence intensity was significantly higher in FN-S than in FN-B $(P=0.0012)$ (Fig. 3c).

A correlation was observed between RI uptake and fluorescence intensity in each lymph node $(P=0.0002$, $\gamma=0.347$ ) (Supplementary Fig. 1). We produced a ROC curve and the AUC of fluorescence intensity in $\mathrm{HN}$ was 0.742 (Supplementary Fig. 2).

Dissecting FNs with fluorescence intensity levels of 1-6 resulted in $92.1 \%$ dissection of HNs (Fig. 4).

As reported by Kinami et al., gastric lymphatic compartments were divided into the following five directions along the main arteries: the left gastric artery area, right gastric artery area, right gastroepiploic area, left gastroepiploic artery area, and posterior gastric artery [8]. Lymphatic basins were found within these lymphatic compartments and were grouped in these five directions. We grouped all $\mathrm{HN}$
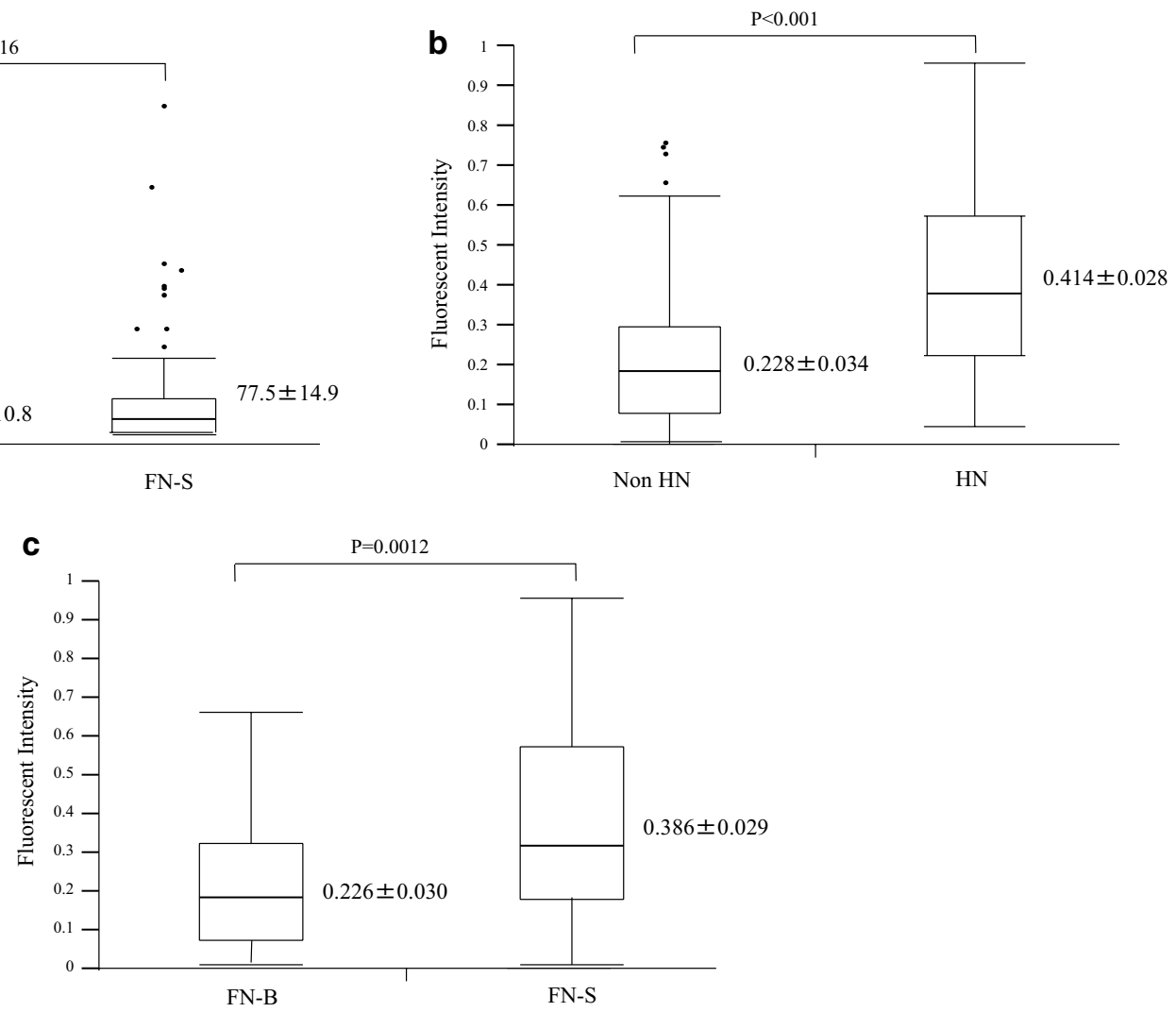

Fig. 3 a The median value of RI uptake by FN-S $(77.5 \pm 14.9)$ was significantly higher than that by FN-B $(32.5 \pm 10.8)(P=0.0016)$. b Median values for fluorescence intensities of $\mathrm{HN}$ and non-HN were $0.414 \pm 0.028$ and $0.228 \pm 0.034$, respectively. Fluorescence intensity was significantly higher in $\mathrm{HN}$ than in non-HN $(P<0.001)$. c Median values for fluorescence intensities of $\mathrm{FN}-\mathrm{S}$ and non-FN-S were $0.386 \pm 0.029$ and $0.226 \pm 0.030$. Fluorescence intensity was significantly higher in FN-S than in FN-B $(P=0.0012)$ 


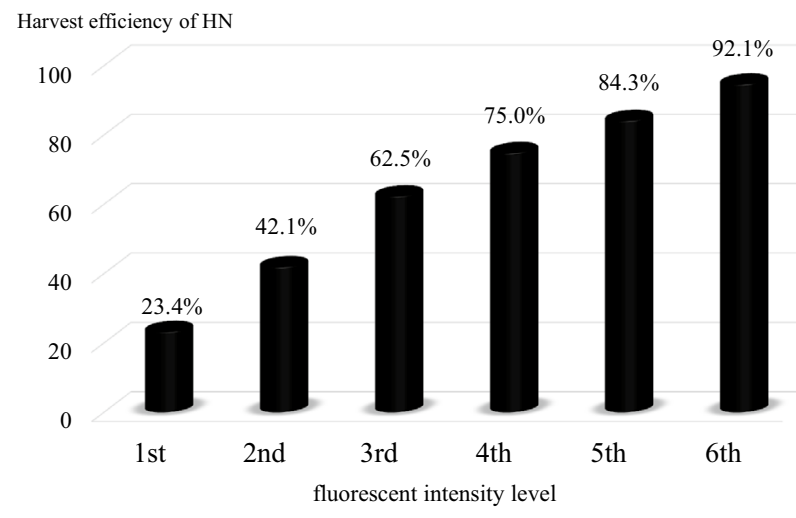

Fig. 4 Harvested rate of HN when fluorescence intensity was used in lymphadenectomy

and FN-S in these five lymphatic basins in each case. The lymphatic basins were consistent between $\mathrm{HN}$ and FN-S at $88.2 \%$ (15 of 17), while those with only FN-S accounted for $11.7 \%$ ( 2 of 17). The lymphatic basins were consistent between $\mathrm{HN}$ and all FNs at $82.3 \%$ (14 of 17), while those with only FN accounted for $17.6 \%$ (3 of 17). No patient had HN outside the FN lymphatic basin.

\section{Discussion}

In the present study, we examined the usefulness of fluorescence intensity in the ICG-based infrared fluorescence method and the safety of SN identification in early gastric cancer. The results revealed a significant relationship between RI uptake and fluorescence intensity. Dissecting lymph nodes with fluorescence intensity levels of 1-6 allowed almost complete dissection of SNs. Accordingly, the ICG fluorescence method may be useful for the distinction of SN using ICG intensity imaging software.

A prospective multicenter trial on SN mapping for gastric cancer $(\mathrm{cT} 1, \mathrm{~T} 2)$ in Japan recently indicated that the dual tracer method was responsible for the higher SN detection rate than that by the radio-guided method alone [10]. However, its general use is limited by the requirement of a radiation control area. Miyashiro et al. reported that the ICG fluorescence imaging system is useful in laparoscopic surgery for gastric cancer [13]. Due to recent advances in the development of devices, ICG-based near-infrared fluorescence can be observed and is useful in revealing SN basins during surgery [15]. We identified FN using HEMS and developed and reported the usefulness of SNNS for breast cancer [3].

In a previous study on ICG fluorescence imaging methods, Tajima et al. reported that $\mathrm{SN}$ were detected in 54 (96.4\%) out of 56 patients. cT1-stage cancers were associated with a significantly higher accuracy rate $(97.2 \%)$ [17]. Kelder et al. demonstrated the clinical usefulness of IREE
(Infrared Ray Electronic Endoscopes) combined with ICG over dye alone to detect illuminated SLN. The SN identification rate and sensitivity for IREE and ICG alone were 99.5 vs $85.8 \%$ and 97.0 vs $48.4 \%$, respectively [7]. ICG nearinfrared fluorescence images were superior to visualization with the naked eye; however, there is currently no method for a quantitative evaluation of fluorescence intensity. Therefore, if we identify a large number of fluorescence lymph nodes, it is difficult to detect SN objectively.

Kelder et al. reported that the SN median numbers by ICG and IREE were $4(0-22)$ and $6(0-34)$ [7]. Tajima et al. showed that the mean number of FN by the ICG fluorescence method was $7.9 \pm 6.1$ in laparoscopy-assisted gastrectomy and 7.2 \pm 6.8 in open gastrectomy [17]. Yoshida et al. found that the ICG fluorescence-positive SN number per patient detected using HEMS was $3.6 \pm 2.1$ [24]. In the present study, the average number of all FNs was 6.52. This number is too high for examinations using H\&E staining during surgery. Since the particle size of ICG is smaller than that of RI, the number of FN detected using HEMS tends to be higher than that during surgical procedures. Therefore, after careful observations by the IRI System, we marked FN with clips and identified FN-S during surgery.

In the present study, we evaluated fluorescence intensity using ICG intensity imaging software after surgery. However, the fluorescence intensity can be determined within a few minutes in an operating room. Therefore, it is useful for quantitative evaluation and FN identification during an operation. Generally the disadvantage of dye methods is the difficulty in detecting nodes in obese patients. ICG fluorescence imaging makes the detection easier than blue dye method. In this study, fluorescence nodes were also identified by a Hyper Eye Medical System and fluorescence intensity was calculated in background. Hence, it is possible that the fluorescence intensity is affected by various conditions similar to the usual ICG method. In this study, although $11 \mathrm{FN}-\mathrm{S}$ could not be detected in $64 \mathrm{HNs}$, no patient had HN outside the FNs lymphatic basin, and for all FNs it was possible to calculate the fluorescence intensity.

It is difficult to define cut-off scores for fluorescence intensity that are common to all cases. HEMS is affected by the background of natural field light. Furthermore, individual differences are observed in fluorescence intensity in each case (Supplementary Table 2). Measurement in a strictly controlled environment is required to achieve a high detection rate. If it is possible to introduce the HEMS system through a trocar, it may be applied to laparoscopic surgery for SNNS and rapidly and accurately measure ICG fluorescence intensity. This paper is a first report of evaluating fluorescence intensity objectively but larger validation studies are necessary for developing the equipment.

When we consider the lymphatic basins of $\mathrm{HN}$ and FN-S in each case, all $\mathrm{HN}$ were present in the lymphatic basin of 
FN-S. This suggests that all SNs could be dissected using fluorescence intensity. Since this study comprised a small patient sample in a single institution, larger validation studies are needed in order to strengthen the results obtained.

\section{Conclusion}

ICG intensity imaging software is more useful for evaluating the fluorescence intensity of FN-S by HEMS in surgery and assessing SN objectively than an RI tracer. SNNS may be conducted even in community hospitals without special equipment if the HEMS system is employed.

\section{Compliance with ethical standards}

Conflict of interest None of the authors has any financial conflicts of interest regarding the study.

Ethical standards All procedures followed were in accordance with the ethical standards of the responsible committee on human experimentation (institutional and national) and with the Helsinki Declaration of 1964 and later versions. The Ethics Committee of Kagoshima University and all patients provided written informed consent to the use of their information.

\section{References}

1. Aikou T, Higashi H, Natsugoe S, Hokita S, Baba M, Tako S. Can sentinel node navigation surgery reduce the extent of lymph node dissection in gastric cancer? Ann Surg Oncol. 2001;8:90S-3S.

2. Ajani JA, Bentrem DJ, Besh S, D'Amico TA, Das P, Denlinger C, et al. Gastric cancer, version 2.2013: featured updates to the NCCN Guidelines. J Natl Compr Cancer Netw. 2013;11:531-46.

3. Aoyama K, Kamio T, Ohchi T, Nishizawa M, Kameoka S. Sentinel lymph node biopsy for breast cancer patients using fluorescence navigation with indocyanine green. World J Surg Oncol. 2011;9:157.

4. Arigami T, Natsugoe S, Uenosono Y, Mataki Y, Ehi K, Higashi H, et al. Evaluation of sentinel node concept in gastric cancer based on lymph node micrometastasis determined by reverse transcription-polymerase chain reaction. Ann Surg. 2006;243:341-7.

5. Giuliano AE, Kirgan DM, Guenther JM, Morton DL. Lymphatic mapping and sentinel lymphadenectomy for breast cancer. Ann Surg. 1994;220:391-8 (Discussion 398-401).

6. Hiratsuka M, Miyashiro I, Ishikawa O, Furukawa H, Motomura K, Ohigashi $\mathrm{H}$, et al. Application of sentinel node biopsy to gastric cancer surgery. Surgery. 2001;129:335-40.

7. Kelder W, Nimura H, Takahashi N, Mitsumori N, van Dam GM, Yanaga K. Sentinel node mapping with indocyanine green (ICG) and infrared ray detection in early gastric cancer: an accurate method that enables a limited lymphadenectomy. Eur J Surg Oncol. 2010;36:552-8.

8. Kinami S, Fujimura T, Ojima E, Fushida S, Ojima T, Funaki H, et al. PTD classification: proposal for a new classification of gastric cancer location based on physiological lymphatic flow. Int $\mathbf{J}$ Clin Oncol. 2008;13:320-9.
9. Kitagawa Y, Fujii H, Mukai M, Kubota T, Ando N, Watanabe $\mathrm{M}$, et al. The role of the sentinel lymph node in gastrointestinal cancer. Surg Clin N Am. 2000;80:1799-809.

10. Kitagawa Y, Takeuchi H, Takagi Y, Natsugoe S, Terashima $\mathrm{M}$, Murakami N, et al. Sentinel node mapping for gastric cancer: a prospective multicenter trial in Japan. J Clin Oncol. 2013;31:3704-10.

11. Kusano M, Tajima Y, Yamazaki K, Kato M, Watanabe M, Miwa $\mathrm{M}$. Sentinel node mapping guided by indocyanine green fluorescence imaging: a new method for sentinel node navigation surgery in gastrointestinal cancer. Digest Surg. 2008;25:103-8.

12. Mitsumori N, Nimura H, Takahashi N, Kawamura M, Aoki H, Shida A, et al. Sentinel lymph node navigation surgery for early stage gastric cancer. World J Gastroenterol. 2014;20:5685-93.

13. Miyashiro I, Miyoshi N, Hiratsuka M, Kishi K, Yamada T, Ohue $\mathrm{M}$, et al. Detection of sentinel node in gastric cancer surgery by indocyanine green fluorescence imaging: comparison with infrared imaging. Ann Surg Oncol. 2008;15:1640-3.

14. Morton DL, Wen DR, Wong JH, Economou JS, Cagle LA, Storm FK, et al. Technical details of intraoperative lymphatic mapping for early stage melanoma. Arch Surg. 1992;127:392-9.

15. Nagao T, Hojo T, Kurihara H, Tsuda H, Tanaka-Akashi S, Kinoshita T. Sentinel lymph node biopsy in breast cancer patients with previous breast augmentation surgery. Breast Cancer. 2014;21:624-8.

16. Nimura H, Narimiya N, Mitsumori N, Yamazaki Y, Yanaga K, Urashima M. Infrared ray electronic endoscopy combined with indocyanine green injection for detection of sentinel nodes of patients with gastric cancer. Br J Surg. 2004;91:575-9.

17. Tajima Y, Yamazaki K, Masuda Y, Kato M, Yasuda D, Aoki T, et al. Sentinel node mapping guided by indocyanine green fluorescence imaging in gastric cancer. Ann Surg. 2009;249:58-62.

18. Takeuchi H, Kitajima M, Kitagawa Y. Sentinel lymph node as a target of molecular diagnosis of lymphatic micrometastasis and local immunoresponse to malignant cells. Cancer Sci. 2008;99:441-50.

19. Uenosono Y, Natsugoe S, Higashi H, Ehi K, Miyazono F, Ishigami S, et al. Evaluation of colloid size for sentinel nodes detection using radioisotope in early gastric cancer. Cancer Lett. 2003;200:19-24.

20. Uenosono Y, Natsugoe S, Ehi K, Arigami T, Hokita S, Aikou T. Detection of sentinel nodes and micrometastases using radioisotope navigation and immunohistochemistry in patients with gastric cancer. Br J Surg. 2005;92:886-9.

21. Yanagita S, Natsugoe S, Uenosono Y, Arigami T, Arima $\mathrm{H}$, Kozono $\mathrm{T}$, et al. Detection of micrometastases in sentinel node navigation surgery for gastric cancer. Surg Oncol. 2008;17:203-10.

22. Yanagita S, Uenosono Y, Arigami T, Daisuke M, Okubo K, Kijima $\mathrm{T}$, et al. The clinical usefulness of the intraoperative detection of sentinel lymph node metastases by a rapid RT-PCR system in patients with gastric cancer. Cancer. 2016;122:386-92.

23. Yano K, Nimura H, Mitsumori N, Takahashi N, Kashiwagi H, Yanaga $\mathrm{K}$. The efficiency of micrometastasis by sentinel node navigation surgery using indocyanine green and infrared ray laparoscopy system for gastric cancer. Gastric Cancer. 2012;15:287-91.

24. Yoshida M, Kubota K, Kuroda J, Ohta K, Nakamura T, Saito $\mathrm{J}$, et al. Indocyanine green injection for detecting sentinel nodes using color fluorescence camera in the laparoscopy-assisted gastrectomy. J Gastroenterol Hepatol. 2012;27(Suppl 3):29-33. 